\title{
New Types of Almost Countable Dense Homogeneous Space
}

\author{
Abdalla Tallafha ${ }^{1}$ and Ahmed Al-Rawashdeh ${ }^{2}$ \\ ${ }^{1}$ Department of Mathematics, Jordan University, Amman 11942, Jordan \\ ${ }^{2}$ Department of Mathematical Sciences, United Arab Emirates University, Al Ain 15551, UAE
}

Correspondence should be addressed to Abdalla Tallafha, a.tallafha@ju.edu.jo

Received 28 September 2009; Revised 19 January 2010; Accepted 9 March 2010

Academic Editor: Richard Wilson

Copyright (C) 2010 A. Tallafha and A. Al-Rawashdeh. This is an open access article distributed under the Creative Commons Attribution License, which permits unrestricted use, distribution, and reproduction in any medium, provided the original work is properly cited.

In 1972, Bennett studied the countable dense homogeneous (CDH) spaces and in 1992, Fitzpatrick, White, and Zhou proved that every $\mathrm{CDH}$ space is a $T_{1}$ space. Afterward Bsoul, Fora, and Tallafha gave another proof for the same result, also they defined the almost $\mathrm{CDH}$ spaces and almost $T_{1}, T_{0}$ spaces, indeed they prove that every ACDH space is an almost $T_{1}$ space. In this paper we introduce a new type of almost $\mathrm{CDH}$ spaces called $\mathrm{ACDH}-1$, we characterize the ACDH spaces, the almost $T_{0}$ spaces, we also give relations between different types of $\mathrm{CDH}$ spaces. We define new type of almost $T_{1}\left(A T_{1}\right)$ spaces, and we study the relations between the old and new definitions. By extending the techniques given by Tallafha, Bsoul, and Fora, we prove that every ACDH-1 is an $A T_{1}$.

\section{Introduction}

In 1920, Sierpinski introduced in [1] the notion of homogeneous spaces, saying that a topological space $X$ is a homogeneous space if for any $x \neq y$ in $X$, then there is a homeomorphism $h$ of $X$ such that $h(x)=y$. Fréchet in [2] and Brouwer in [3] observed that the $n$-dimensional Euclidean space $\mathbb{R}^{n}$ has the property that if $A, B$ are countable dense subsets of $\mathbb{R}^{n}$, then there is a homeomorphism $h$ of $\mathbb{R}^{n}$ such that $h(A)=B$. Afterward, in 1972 the abstract study was begun by Bennett in [4], who called such spaces the countable dense homogeneous $(\mathrm{CDH})$ spaces.

In 1974, Lauer defined in [5] the densely homogeneous (DH) spaces, and in 1992, Fitzpatrick et al. proved in [6] that $\mathrm{DH}$ and $\mathrm{CDH}$ spaces are $T_{1}$ spaces, and afterward Tallafha et al. in [7] gave another proof for the fact that $\mathrm{CDH}$ spaces are $T_{1}$ spaces. In [8], Fora et al. defined almost $\mathrm{CDH}(\mathrm{ACDH})$ spaces, almost $T_{0}, T_{1}\left(A T_{0}, A T_{1}\right)$ spaces, and they discussed the relation between such spaces. 
In the first part of this paper, we introduce the definitions given by Fora et al. in [8] for almost $\mathrm{CDH}$ spaces, almost $T_{0}, T_{1}$ spaces. We give a characterization of almost $\mathrm{CDH}$ spaces besides we define a new type of almost $\mathrm{CDH}$ (ACDH-1) spaces, Transposition Homogeneous space $(\mathrm{TH})$. Also, we discuss the relation between the new type and the others.

In Section 4 we give a characterization of almost $T_{0}$ we introduce new definition of almost $T_{1}$ space $A S T_{1}$, and we study the relation between them and the old ones. We finally prove our main result using the idea of almost closurely ordered sets, more precisely, we show that every almost $\mathrm{CDH}$ space of type 1 (ACDH-1) is an $A S T_{1}$ space.

Finally, the following abbreviations and symbols will be used throughout this paper.

For a subset $A$ of a topological space $(X, \tau)$, we write $\bar{A}$, or $\mathrm{Cl}(A)$ for the closure of $A$, for $x \in X$, by $\bar{x}$ which denotes the closure of $\{x\}$ and $|A|$ which denotes the cardinality of $A$. By $H(X)$ we mean the set of all homeomorphisms of $X, \Delta$ refers to the symmetric difference of sets, $\left(X, \tau_{\text {ind }}\right),\left(X, \tau_{\text {dis }}\right),\left(X, \tau_{\text {cof }}\right)$, and $\left(X, \tau_{\text {coc }}\right)$ denote the set $X$ with the indiscrete, discrete, cofinite, and cocountable topologies, and $\left(\mathbb{R}, \tau_{l \cdot r}\right),\left(\mathbb{R}, \tau_{r \cdot r}\right)$, and $\left(\mathbb{R}, \tau_{u}\right)$ denote $\mathbb{R}$, with the left ray, right ray, and the usual topologies, respectively.

\section{Almost CDH Spaces}

Fora et al. in [8] defined almost and strong almost countable dense homogeneous spaces.

Definition 2.1 (see [8]). A space $(X, \tau)$ is called an almost countable dense homogeneous $(\mathrm{ACDH})$ space if it is a separable space and for any two countable dense subsets $K_{1}, K_{2}$, there are two finite subsets $F_{1}, F_{2}, K_{1} \cap F_{1}=K_{2} \cap F_{2}=\emptyset$ and $h \in H(X)$ such that $h\left(K_{1} \cup F_{1}\right)=K_{2} \cup F_{2}$ and $h\left(F_{1}\right) \cap F_{2}=\emptyset$. In addition, if $\left|F_{1}\right|=\left|F_{2}\right|$, then $(X, \tau)$ is called a strong ACDH space which is denoted by SACDH.

Remark 2.2. In the previous definition the condition $h\left(F_{1}\right) \cap F_{2}=\emptyset$ is redundant since if there exist finite sets $B_{1}$ and $B_{2}$ such that $K_{1} \cap B_{1}=K_{2} \cap B_{2}=\emptyset$ and $h\left(K_{1} \cup B_{1}\right)=K_{2} \cup B_{2}$, then we choose the finite sets $F_{1}$ and $F_{2}$ as follows $F_{1}=B_{1} \backslash h^{-1}(C), F_{2}=B_{2} \backslash C$, where $C=h\left(B_{1}\right) \cap B_{2}$.

Theorem 2.3 (see [8]). If $X$ is countable and $(X, \tau)$ is $S A C D H$, then $\tau$ is the discrete topology.

Let $(X, \tau)$ be any topological space. For $x \in X$, let $A_{x}=\{y \in X: \bar{y}=\bar{x}\}, A=\{x:$ $\left.\left|A_{x}\right|>1\right\}, B=\left\{x:|\bar{x}| \geq \aleph_{0}\right\}, C_{x}=\{y \in X: x \in \bar{y}\}$, and $C=\left\{x:\left|C_{x}\right| \geq \aleph_{0}\right\}$. And Let $F=\{x \in X:\{x\}$ is not a closed set $\}$.

Definition 2.4 (see [8]). A topological space $(X, \tau)$ is called almost $T_{o}\left(A T_{0}\right)$ if $|A|<\aleph_{0}$. If, in addition, $|B|<\aleph_{0}$, then $(X, \tau)$ is called strong almost $T_{o}\left(S A T_{o}\right)$.

Clearly every $T_{o}$-space is $A T_{o}$.

Definition 2.5 (see [8]). A topological space $(X, \tau)$ is called almost $T_{1}$ and denoted by $A T_{1}$ if it is $S A T_{o}$ and $|C|<\aleph_{0}$, that is, $A, B$, and $C$ are all finite sets.

Definition 2.6 (see [8]). A topological space $(X, \tau)$ is called strong almost $T_{1}$ space $\left(S A T_{1}\right)$ if $|F|<\aleph_{0}$. Clearly every $T_{1}$ space is $S A T_{1}$ and every $S A T_{1}$ is $A T_{1}$.

Theorem 2.7 (see [8]). Every ACDH space is $A T_{0}$.

Theorem 2.8 (see [8]). Let $(X, \tau)$ be ACDH space. If $|X| \leq \aleph_{0}$, then $(X, \tau)$ is $A T_{1}$. 
Now let us define a new type of almost $\mathrm{CDH}$ spaces.

Definition 2.9. A topological space $(X, \tau)$ is called an almost $\mathrm{CDH}$ of type 1 (ACDH-1) if it is separable space and there exists a finite subset $F$ such that, for any two countable dense subsets $A$ and $B$ of $X$, there exists $h \in H(X)$ such that $h(A \backslash F)=B \backslash F$. Note that from now on, we will refer to $\mathrm{F}$ by a related finite set. result.

Clearly every finite space is an almost $\mathrm{CDH}$ of type 1 . Moreover, we have the following

Proposition 2.10. If $(X, \tau)$ is $A C D H-1,|X| \leq \aleph_{0}$, and $F$ is a related finite set, then for all $x \notin F$, we have $\{x\}$ which is an open set in $X$.

Proof. Let $x \in X \backslash F$. If $\{x\}$ is not an open set in $X$, then $\overline{X \backslash\{x\}}=X$, therefore there is $h \in H(X)$ such that $h(X \backslash F)=(X \backslash\{x\}) \backslash F$ which is a contradiction.

As an application of Proposition 2.10, we have the following corollary.

Corollary 2.11. If $(X, \tau)$ is an $A C D H-1,|X| \leq \aleph_{0}$, then there exists the smallest related finite set $A$.

Proof. Let $(X, \tau)$ be a nondiscrete ACDH-1 space and $A=\{x \in X:\{x\}$ not open $\}$. As $\tau \neq \tau_{\text {dis, }}$, $A \neq \phi$. Also by Proposition 2.10, $A \subseteq F$, for all related finite sets $F$, so $A$ is finite. If $K_{1}, K_{2}$ are two dense sets and $h \in H(X)$, then $X \backslash A \subseteq K_{1} \cap K_{2}$ and $h(X \backslash A)=X \backslash A$, hence $h\left(K_{1} \backslash A\right)=h(X \backslash A)=X \backslash A=K_{2} \backslash A$; therefore $A$ is the smallest finite related set. Moreover, if $\tau=\tau_{\text {dis }}$, then $A=\emptyset$.

The following example shows that $\mathrm{ACDH}-1$ space need be a $\mathrm{CDH}$ space.

Example 2.12. Let $X=\mathbf{N}$ and $\beta=\{\{1,2\},\{3,4\},\{5\},\{6\}, \ldots\}$. Let $A, B$ be two dense subsets of $X$. Then $\{5,6,7, \ldots\} \subseteq A \cap B$ let $F=\{1,2,3,4\}$, hence $(X, \tau(\beta))$ is ACDH-1 space which is not a $\mathrm{CDH}$ space as it is not a $T_{1}$ space.

Now let prove the following characterizations of ACDH spaces.

Theorem 2.13. If $(X, \tau)$ is a separable space, then the following are equivalent

(i) $(X, \tau)$ is an ACDH space

(ii) For any two countable dense subsets $K_{1}, K_{2}$, there exist two finite subsets $F_{1}, F_{2}$ of $K_{1}, K_{2}$, respectively, and $h \in H(X)$ such that $h\left(K_{1} \backslash F_{1}\right)=K_{2} \backslash F_{2}$.

(iii) For any two countable dense subsets $K_{1}, K_{2}$, there exist two equipotent finite subsets $F_{1}, F_{2}$, and $h \in H(X)$ such that $h\left(K_{1} \backslash F_{1}\right)=K_{2} \backslash F_{2}$.

(iv) For any two countable dense subsets $K_{1}, K_{2}$, there exist two finite subsets $F_{1}, F_{2}$, and $h \in$ $H(X)$ such that $h\left(K_{1} \backslash F_{1}\right)=K_{2} \backslash F_{2}$.

Proof. (i) implies (ii) Suppose that $(X, \tau)$ is ACDH space. Let $K_{1}, K_{2}$ be two countable dense sets. Then there exist two finite sets $G_{1}, G_{2}$ and $h \in H(X)$ such that $G_{1} \cap K_{1}=G_{2} \cap K_{2}=$ $G_{2} \cap h\left(G_{1}\right)=\emptyset$ and $h\left(K_{1} \cup G_{1}\right)=K_{2} \cup G_{2}$. Let $F_{1}=h^{-1}\left(G_{2}\right)$ and $F_{2}=h\left(G_{1}\right)$, so $F_{1}, F_{2}$ are two finite sets and

$$
h\left(K_{1} \backslash F_{1}\right)=\left(h\left(K_{1} \cup G_{1}\right) \backslash h\left(G_{1}\right)\right) \backslash G_{2}=K_{2} \backslash F_{2} .
$$


(ii) implies (iii) Let $K_{1}, K_{2}$ be two countable dense sets, then there exist two finite sets $F_{1}, F_{2}$, and $h \in H(X)$ such that $F_{1} \subseteq K_{1}, F_{2} \subseteq K_{2}$, and $h\left(K_{1} \backslash F_{1}\right)=K_{2} \backslash F_{2}$. Let $G_{1}=F_{1} \cup h^{-1}\left(F_{2}\right)$ and $G_{2}=h\left(F_{1}\right) \cup F_{2}$. Clearly $\left|G_{1}\right|=\left|G_{2}\right|$. Moreover,

$$
h\left(K_{1} \backslash G_{1}\right)=h\left(K_{1} \backslash F_{1} \cup h^{-1}\left(F_{2}\right)\right)=K_{2} \backslash F_{2} .
$$

But clearly $\left(h\left(F_{1}\right) \backslash F_{2}\right) \cap K_{2}=\emptyset$, so $K_{2} \backslash F_{2}=K_{2} \backslash G_{2}$. (iii) implies that (iv) is clear.

(iv) implies (i) Let $K_{1}, K_{2}$ be two countable dense sets, then there are $G_{1}, G_{2}$ finite sets and $h \in H(X)$ such that $h\left(K_{1} \backslash G_{1}\right)=K_{2} \backslash G_{2}$.

Let $F_{1}=h^{-1}\left(G_{2} \cap K_{2}\right) \backslash\left(G_{1} \cap K_{1}\right)$ and $F_{2}=h\left(G_{1} \cap K_{1}\right) \backslash\left(G_{2} \cap K_{2}\right)$. Then $F_{1}, F_{2}$ are two finite sets. If $t \in F_{1} \cap K_{1}$, then $t \in K_{1} \backslash G_{1}$ and $h(t) \in G_{2} \cap K_{2}$ which gives a contradiction. Similarly, $F_{2} \cap K_{2}=\emptyset$. Moreover,

$$
\begin{aligned}
h\left(K_{1} \cup F_{1}\right) & =h\left(K_{1}\right) \cup\left[\left(G_{2} \cap K_{2}\right) \backslash h\left(G_{1} \cap K_{1}\right)\right] \\
& =h\left(K_{1}\right) \cup\left(G_{2} \cap K_{2}\right) \\
& =h\left(\left(K_{1} \backslash G_{1}\right) \cup\left(K_{1} \cap G_{1}\right)\right) \cup\left(G_{2} \cap K_{2}\right) \\
& =K_{2} \backslash G_{2} \cup h\left(K_{1} \cap G_{1}\right) \cup\left(G_{2} \cap K_{2}\right) \\
& =K_{2} \cup\left(h\left(K_{1} \cap G_{1}\right) \backslash K_{2}\right) \\
& =K_{2} \cup F_{2} .
\end{aligned}
$$

Consequently, we have the following result.

Corollary 2.14. Every ACDH-1 space is an ACDH space.

Theorem 2.15. Let $(X, \tau)$ be a separable space. Then $(X, \tau)$ is an SACDH space if and only if for every two countable dense sets $K_{1}, K_{2}$, there exist two equipotent finite subsets $F_{1}, F_{2}$ of $K_{1}, K_{2}$, respectively, and $h \in H(X)$ such that $h\left(K_{1} \backslash F_{1}\right)=K_{2} \backslash F_{2}$.

Proof. Let $K_{1}, K_{2}$ be two countable dense sets, so there exist $h \in H(X), G_{1} \subseteq K_{1}, G_{2} \subseteq K_{2}$, and $\left|G_{1}\right|=\left|G_{2}\right|<\aleph_{0}$ such that $h\left(K_{1} \backslash G_{1}\right)=K_{2} \backslash G_{2}$. Let $F_{1}=h^{-1}\left(G_{2}\right) \backslash K_{1}$ and $F_{2}=h\left(G_{1}\right) \backslash K_{2}$. It is clear that $F_{1} \cap K_{1}=F_{2} \cap K_{2}=\emptyset$. Also,

$$
\begin{aligned}
\left|F_{1}\right| & =\left|h^{-1}\left(G_{2}\right) \backslash K_{1}\right| \\
& =\left|h^{-1}\left(G_{2}\right) \backslash\left(K_{1} \cap h^{-1}\left(G_{2}\right)\right)\right| \\
& =\left|h^{-1}\left(G_{2}\right)\right|-\left|K_{1} \cap h^{-1}\left(G_{2}\right)\right| \\
& =\left|G_{1}\right|-\left|\left(h\left(K_{1} \backslash G_{1}\right) \cup h\left(G_{1}\right)\right) \cap G_{2}\right| \\
& =\left|G_{1}\right|-\left|h\left(G_{1}\right) \cap G_{2}\right| \\
& =\left|G_{1}\right|-\left|G_{2} \cap h\left(G_{1}\right) \cup\left(\left(K_{2} \backslash G_{2}\right) \cap h\left(G_{1}\right)\right)\right| \\
& =\left|h\left(G_{1}\right)\right|-\left|K_{2} \cap h\left(G_{1}\right)\right| \\
& =\left|F_{2}\right| .
\end{aligned}
$$


Moreover,

$$
\begin{aligned}
h\left(K_{1} \cup F_{1}\right) & =h\left(K_{1}\right) \cup h\left(F_{1}\right) \\
& =h\left(K_{1}\right) \cup\left(G_{2} \backslash h\left(K_{1}\right)\right) \\
& =h\left(K_{1}\right) \cup G_{2} \\
& =h\left(\left(K_{1} \backslash G_{1}\right) \cup G_{1}\right) \cup G_{2} \\
& =K_{2} \cup h\left(G_{1}\right) \\
& =K_{2} \cup\left(h\left(G_{1}\right) \backslash K_{2}\right) \\
& =K_{2} \cup F_{2} .
\end{aligned}
$$

Hence, $(X, \tau)$ is an SACDH space.

Conversely, assume that $(X, \tau)$ is an SACDH space. Let $K_{1}, K_{2}$ be two countable dense sets, therefore, there are two finite sets $G_{1}, G_{2}$ with $\left|G_{1}\right|=\left|G_{2}\right|$ and $h \in H(X)$ such that $h\left(K_{1} \cup\right.$ $\left.G_{1}\right)=K_{2} \cup G_{2}$; moreover, $K_{1} \cap G_{1}=G_{2} \cap K_{2}=h\left(G_{1}\right) \cap G_{2}=\emptyset$. Let $F_{1}=h^{-1}\left(G_{2}\right)$ and $F_{2}=h\left(G_{1}\right)$. Then $\left|F_{1}\right|=\left|F_{2}\right|$, moreover, $F_{1} \subseteq K_{1}$ and $F_{2} \subseteq K_{2}$. We need to prove that $h\left(K_{1} \backslash F_{1}\right)=K_{2} \backslash F_{2}$. Now, $h\left(K_{1} \backslash F_{1}\right)=h\left(K_{1} \backslash h^{-1}\left(G_{2}\right)\right)=h\left(K_{1}\right) \backslash G_{2}$. Claim that $h\left(K_{1}\right) \backslash G_{2}=K_{2} \backslash F_{2}$. If $x \in h\left(K_{1}\right) \backslash G_{2}$, then $x \in\left(K_{2} \cup G_{2}\right) \backslash G_{2}=K_{2}$. As $K_{1} \cap G_{1}=\emptyset, x \notin h\left(G_{1}\right)=F_{2}$, so $x \in K_{2} \backslash F_{2}$. To prove the other inclusion, suppose that $x \in K_{2} \backslash F_{2}=K_{2} \backslash h\left(G_{1}\right)=h\left(h^{-1}\left(K_{2}\right) \backslash G_{1}\right)$. Therefore, $x=h(t)$, for some $t \in h^{-1}\left(K_{2}\right) \backslash G_{1}$, and then $t \in\left(K_{1} \cup G_{1}\right) \backslash G_{1}$ so $t \in K_{1}$. Also $t \notin h^{-1}\left(G_{2}\right)$, hence $x \in h\left(K_{1}\right) \backslash G_{2}$.

Consequently, we have the following result.

Corollary 2.16. Every ACDH-1 space is SACDH.

\section{T-Homogeneous Spaces}

A transposition on $X$ is a permutation on $X$ which exchanges the places of two elements $x, y$, while leaving all the other elements unchanged. Now we will define the TranspositionHomogeneous (TH) spaces, and we will show that every TH SACDH space is a CDH space.

Definition 3.1. A space $(X, \tau)$ is called Transposition-Homogeneous (TH) space if every transposition on $\mathrm{X}$ is a homeomorphism.

Proposition 3.2. A space $(X, \tau)$ is $(T H)$ if and only if, for any two finite subsets $F_{1}, F_{2}$ with the same cardinality and for every $h \in H(X)$, there exists $h^{\prime} \in H(X)$ such that

(1) $h^{\prime}\left(F_{1}\right)=F_{2}$,

(2) $h^{\prime}(x)=h(x)$, for every $x \notin F_{1} \cup h^{-1}\left(F_{2}\right)$,

(3) $h^{\prime}\left(h^{-1}\left(F_{2}\right)\right)=h\left(F_{1}\right)$.

Proof. Let $(X, \tau)$ be a TH space and $F$ a finite subset of $X$. Let $\sigma$ be a permutation which fixes $X \backslash F$, clearly $\sigma$ is a composition of finite transpositions which is a homeomorphism. Let 
$F_{1}, F_{2}$ be two finite subsets with the same cardinality and $h \in H(X)$, clearly $\left|F_{1} \backslash h^{-1}\left(F_{2}\right)\right|=$ $\left|h^{-1}\left(F_{2}\right) \backslash F_{1}\right|$ and $F=\left(F_{1} \backslash h^{-1}\left(F_{2}\right)\right) \sqcup\left(h^{-1}\left(F_{2}\right) \backslash F_{1}\right)$ are a disjoint union of finite sets which is finite. Let $f: F \rightarrow F$ be a bijection such that $f\left(F_{1} \backslash h^{-1}\left(F_{2}\right)\right)=h^{-1}\left(F_{2}\right) \backslash F_{1}$. For each $x \in F_{1} \backslash h^{-1}\left(F_{2}\right)$, let $\sigma_{x}$ be a transposition on $X$ which transposes $x$ and $f(x)$. Now let $\sigma$ be the finite composition of the transpositions $\sigma_{x}, x \in F_{1} \backslash h^{-1}\left(F_{2}\right)$. Then $\sigma_{x}(y)=y$ for all $y \in X \backslash F, \sigma\left(F_{1}\right)=h^{-1}\left(F_{2}\right)$, and $\sigma\left(h^{-1}\left(F_{2}\right)\right)=F_{1}$. Now $h^{\prime}=h \circ \sigma$ is the required function since $h^{\prime}(x)=h(x)$, for every $x \in X \backslash F$.

The converse is obvious by choosing $h$ to be the identity and $F_{1}=\{x\}, F_{2}=\{y\}$.

Example 3.3. One can show that the spaces $\left(X, \tau_{\text {ind }}\right),\left(X, \tau_{\text {dis }}\right),\left(X, \tau_{\text {cof }}\right)$, and $\left(X, \tau_{\text {coc }}\right)$ are all $\mathrm{TH}$ spaces. However, the spaces $\left(\mathbb{R}, \tau_{l . r}\right)$ and $\left(\mathbb{R}, \tau_{u}\right)$ are not $\mathrm{TH}$ spaces.

The following example shows that ACDH-1, TH space need be CDH space, hence ACDH TH space need be a CDH space.

Example 3.4. Let $X$ be such that $1<|X|<\aleph_{0}$, with the indiscrete topology. The space $(X, \tau)$ is a TH-space and it is also ACDH-1, but it is not a $\mathrm{CDH}$ space as it is not a $T_{1}$ space.

Theorem 3.5. If $(X, \tau)$ is $S A C D H, T H$ space, then $(X, \tau)$ is a CDH space.

Proof. If $A, B$ are two countable dense subsets of $X$, then there exist two finite subsets $F_{1}, F_{2}$ of $A, B$, respectively with $\left|F_{1}\right|=\left|F_{2}\right|$ and there is $h \in H(X)$ such that $h\left(A \backslash F_{1}\right)=B \backslash F_{2}$. As $X$ is a TH space, there exists $h^{\prime} \in H(X)$ such that $h^{\prime}\left(F_{1}\right)=F_{2}, h^{\prime}(x)=h(x)$, for all $x \notin F_{1} \cup h^{-1}\left(F_{2}\right)$ and $h^{\prime}\left(h^{-1}\left(F_{2}\right)\right)=h\left(F_{1}\right)$. To show that $h^{\prime}(A)=B$ we show first that $A \cap h^{-1}\left(F_{2}\right) \subseteq F_{1}$. Suppose that there is $x \in\left(A \cap h^{-1}\left(F_{2}\right)\right) \backslash F_{1}$. Then $h(x) \in B \backslash F_{2}$ which gives a contradiction. Hence $h^{\prime}\left(A \backslash F_{1}\right)=h\left(A \backslash F_{1}\right)=B \backslash F_{2}$, so $h^{\prime}(A)=h^{\prime}\left(\left(A \backslash F_{1}\right) \cup F_{1}\right)=\left(B \backslash F_{2}\right) \cup F_{2}=B$.

Let $(X, \tau)$ be an ACDH-1 space and $\mathfrak{F}=\{F: F$ is a related set $\}$ and define $D=\{A \subseteq$ $X: A$ is acountable dense subset of $X\}$, and also we define the relation $\sim$ on $D$ by: $A \sim B$ if and only if there is $F \in \mathfrak{F}$ such that $|A \cap F|=|B \cap F|$. Let $D_{1}=\{(A, B) \in D \times D: A \sim B\}$.

Now we have the following result.

Theorem 3.6. If $(X, \tau)$ is an $A C D H-1, T H$ space, and $(A, B) \in D_{1}$, then there is $h \in H(X)$ such that $h(A)=B$.

Proof. Suppose that $(A, B) \in D_{1}$, so there are $F \in \mathfrak{F}$ and $h \in H(X)$ such that $h(A \backslash F)=$ $B \backslash F$ with $|A \cap F|=|B \cap F|$. If $A \cap F=\emptyset$, then we are done. In general let $F_{1}=F \cap A$ and $F_{2}=F \cap B$, therefore $\left|F_{1}\right|=\left|F_{2}\right|$. As $X$ is a TH space, there exists $h^{\prime} \in H(X)$ such that $h^{\prime}\left(F_{1}\right)=$ $F_{2}$ and $h^{\prime}(x)=h(x)$, for all $x \notin F_{1} \cup h^{-1}\left(F_{2}\right)$ and $h^{\prime}\left(h^{-1}\left(F_{2}\right)\right)=h\left(F_{1}\right)$, also $A \cap h^{-1}\left(F_{2}\right) \subseteq F$. Now $h^{\prime}\left(A \backslash \mathrm{F}_{1}\right)=h^{\prime}(A \backslash F)=h(A \backslash F)=B \backslash F=B \backslash F_{2}$, so $h^{\prime}(A)=h^{\prime}\left(\left(A \backslash F_{1}\right) \cup F_{1}\right)=\left(B \backslash F_{2}\right) \cup F_{2}=B$.

Consequently, we have the following Corollary.

Corollary 3.7. If $(X, \tau)$ is an ACDH-1, TH space, and $D_{1}=D \times D$, then $(X, \tau)$ is a CDH space.

\section{Almost CDH Spaces, and New Separation Axioms}

We know that almost $\mathrm{CDH}$ space is not a $T_{0}$ space. In this section we will give a characterization of almost $T_{0}$ space, also we will give a new definition of almost $T_{1}$ space. 
Theorem 4.1. Let $(X, \tau)$ be a topological space. Then $(X, \tau)$ is $A T_{0}$ space if and only if there exists a finite subset $F$ of $X$, such that, for all $x \neq y$ and $\{x, y\} \cap F=\emptyset$, there is an open set containing only one of $x, y$. We will refer to $F$ by a related finite set $F$.

Proof. Assume that $(X, \tau)$ is an $A T_{0}$ space. Let $F=A$. Then for all $x \neq y$ with $\{x, y\} \cap F=\emptyset$, we have that $\left|A_{x}\right|=1$ and $\left|A_{y}\right|=1$, hence $y \notin \bar{x}$ or $x \notin \bar{y}$. Conversely, suppose that there exists a finite set $F$ such that for all $x \neq y$ with $\{x, y\} \cap F=\emptyset$, there is an open set containing only one of $x, y$. If $|A|$ is an infinite, then there exists a denumerable subset of $A$, say $\left\{x_{1}, x_{2}, \ldots\right\}$, and $\left|A_{x_{n}}\right|>1$, for all $n \in \mathbb{N}$, so there exist $y_{n} \in A_{x_{n}}$ and $y_{n} \neq x_{n}$ for all $n \in \mathbb{N}$. Therefore there is $n_{0} \in \mathbb{N}$ such that $x_{n_{0}} \neq y_{n_{0}}$ are both not in $F$ and $\bar{x}_{n_{0}}=\bar{y}_{n_{0}}$, which gives a contradiction.

Definition 4.2. A space $(X, \tau)$ is called an almost strong $T_{1}\left(A S T_{1}\right)$ space if there is a finite subset $F$ of $X$ such that, for all $x \neq y$ and $\{x, y\} \cap F=\emptyset$, there are two open subsets $u_{1}, u_{2}$ of $X$, such that $x \in u_{1} \backslash u_{2}$ and $y \in u_{2} \backslash u_{1} . F$ is called the related finite set.

One may easily prove the following proposition.

Proposition 4.3. Let $(X, \tau)$ be a topological space. If $(X, \tau)$ is an $A S T_{1}$ space, then for all $x \notin F$, we have $\bar{x} \subseteq\{x\} \cup F$; where $F$ is a related finite set. Conversely, if there is a finite set $F$ such that for all $x \notin F, \bar{x} \subseteq\{x\} \cup F$, then $(X, \tau)$ is an $A S T_{1}$.

In the following results we show that the new separation axiom $A S T_{1}$ is stronger than the one defined by Fora et al. in [8].

Proposition 4.4. Every $A S T_{1}$ space is $A T_{1}$ space.

Proof. Suppose that $(X, \tau)$ is an $A S T_{1}$ space, and let $F$ be a related finite set. By Theorem 4.1 it is an $A T_{0}$, therefore, $|A|<\aleph_{0}$. By Proposition 4.3, for all $x \notin F$, we have $\bar{x} \subseteq\{x\} \cup F$, so that $B \subseteq F$. If $|C| \geq \aleph_{0}$, then there is $x_{n} \in C$ such that for all $m \neq n, x_{n} \neq x_{m}$ and $\left|C_{x_{n}}\right| \geq \aleph_{0}$, for all $n \in \mathbb{N}$. Let $n_{1}$ be such that $x_{n_{1}} \notin F$. Therefore $\left|C_{x_{n_{1}}}\right| \geq \aleph_{0}$, then there is $t \in C_{x_{n_{1}}} \backslash F$, so $x_{n_{1}} \in \bar{t}$, which gives a contradiction, and hence the proposition is proved. be true.

The following example shows that the converse of the previous proposition need not

Example 4.5. Let $X=\mathbb{N},: \beta=\{\{1\},\{1,2\},\{3\},\{3,4\},\{5\},\{5,6\}, \ldots\}$ so $\beta$ is a base for some topology on $X$. Note that for $n \in \mathbb{N}$, we have

$$
\bar{n}= \begin{cases}\{n, n+1\} ; & n \text { is odd } \\ \{n\} ; & n \text { is even. }\end{cases}
$$

Therefore, for all $x \in \mathbb{N}, A_{x}=\{x\}$, hence $A=\emptyset, B=\emptyset$ as for all $n \in \mathbb{N},|\bar{n}| \leq 2$. Now for $n \in \mathbb{N}$

$$
C_{n}= \begin{cases}\{n-1, n\} ; & n \text { is even } \\ \{n\} ; & n \text { is odd }\end{cases}
$$


Therefore, $C=\emptyset$, and then $(X, \tau(\beta))$ is $A T_{1}$ space. Let $F$ be any finite subset of $X$. Let $m=$ sup : $F$, as $2 m+2 \in \mathrm{Cl}\{2 m+1\}$ and $2 m+2,2 m+1$ are both not in $F,(X, \tau(\beta))$ is not an $A S T_{1}$ space.

One may easily prove the following proposition.

Proposition 4.6. Every $S A T_{1}$ space is $A S T_{1}$ space.

Fitzpatrick et al. proved in [6] that every $\mathrm{CDH}$ space is a $T_{1}$ space. Indeed, Tallafha et al. in [7] gave us another proof for the same argument by using the idea of closurely ordered sets. Now, we will prove that every ACDH-1 space is $A S T_{1}$ space by using the idea of almost closurely ordered sets.

Definition 4.7 (see [7]). Let $(X, \tau)$ be a topological space. A countable subset $K$ of $X$ is said to have the closurely ordered property if there exists a numeration of $K$, say $K=\left\{x_{1}, x_{2}, \ldots\right\}$ such that for all $n \geq 2, x_{n} \notin \mathrm{Cl}\left\{x_{1}, x_{2}, \ldots, x_{n-1}\right\}$. The numeration $\left\{x_{1}, x_{2}, \ldots\right\}$ is called closurely ordered countable set.

Definition 4.8 (see [7]). A countable collection $\mathfrak{A}$ of subsets of $X$ is said to have the closurely ordered countable property if $\mathfrak{A}$ can be written as $\mathfrak{A}=\left\{A_{1}, A_{2}, \ldots\right\}$, where $A_{n} \cap \operatorname{Cl}\left\{\bigcup_{i=1}^{n-1} A_{i}\right\}=$ $\emptyset$. The form $\left\{A_{1}, A_{2}, \ldots\right\}$ is called closurely ordered countable family.

Theorem 4.9 (see [7]). Let $(X, \tau)$ be a topological space and let $K$ be any countable dense subset of $X$. Then there exists a countable dense subset $K_{1}$ of $K$, such that $K_{1}$ is closurely ordered countable set.

Theorem 4.10 (see [7]). Let $(X, \tau)$ be a topological space, then,

(i) if $h: X \rightarrow Y$ is an injective open function and $K$ has the closurely ordered property in $X$, then $h(K)$ has the closurely ordered property in $Y$,

(ii) having closurely ordered property, is a topological property,

(iii) every subset of a set having closurely ordered property must have closurely ordered property.

Now let us define the following.

Definition 4.11. A countable set $K$ in $(X, \tau)$ is said to have the almost closurely ordered property if there is a finite set $F$ in $X$ such that $K \backslash F$ has the closurely ordered property. If $K \backslash F=\left\{x_{1}, x_{2}, \ldots\right\}$ is a closurely ordered set, then $K$ is called almost closurely ordered set.

Proposition 4.12. If $(X, \tau)$ is an $A S T_{1}$ space and $F$ is a related finite set, then each doubleton $\{x, y\} \subseteq$ $X \backslash F$ has the closurely ordered property. Conversely, in a topological space $(X, \tau)$ if there exists a finite set $F$ all doubletons $\{x, y\} \subseteq X \backslash F$ have the closurely ordered property, then $(X, \tau)$, is an $A T_{0}$ space.

Proof. The first part is clear. To prove the converse, assume that there is such a finite set $F$. Let $x, y$ be such that $x \neq y$ and $\{x, y\} \subseteq X \backslash F$. So $x \notin \bar{y}$ or $y \notin \bar{x}$, by Theorem $4.1(X, \tau)$ is an $A T_{0}$.

Theorem 4.13. Every ACDH-1 space is $A T_{0}$ space.

Proof. Assume that $(X, \tau)$ is an ACDH-1 space and $F$ is a related finite set. We want to show that $F$ is the desired set. If $x \neq y$ with $\{x, y\} \cap F=\emptyset$ and $K$ is a countable dense subset of $X$, by Theorem 4.9 , we may assume that $K$ has the closurely ordered property, also by 
Theorem 4.10, $K \backslash F$ has the closurely ordered property. Now $\{x, y\} \cup K$ is also a countable dense subset of $X$, therefore there is $h \in H(X)$ such that $h(K \backslash F)=(K \cup\{x, y\}) \backslash F$. So $\{x, y\}$ has the closurely ordered property, the result follows by Theorem 4.10.

Theorem 4.14 (see [7]). If $(X, \tau)$ a topological space and $K$ is a countable dense subset, then there exists a countable collection of subsets $A_{1}, A_{2}, \ldots$ of $K$ such that

(i) $A=\bigcup_{n=1}^{m} A_{n} \subseteq K: m \leq \aleph_{0}$,

(ii) $\left\{A_{1}, A_{2}, \ldots\right\}$ is closurely ordered countable family,

(iii) each $A_{k}$ has the closurely ordered property,

(iv) each $A_{k}$ is either a singleton or an infinite set,

(v) $\bar{A}=\bar{K}$,

(vi) if $A_{k}$ is a singleton, say $\left\{a_{k}\right\}$, then $a_{k} \notin \bar{x}$ and $x \notin C l\left\{a_{k}\right\}$, for all $x \in A \backslash\left\{a_{k}\right\}$,

(vii) if $A_{k}=\left\{a_{1}^{k}, a_{2}^{k}, \ldots\right\}$ is infinite set, then $a_{i}^{k} \in C l\left\{a_{1+i}^{k}\right\}$, for all $i$.

It is easy to prove the following result.

Proposition 4.15. The properties (i)-(vii) in the last theorem are all preserved under homeomorphisms.

We now prove the following theorem, that will be used to prove our main result.

Theorem 4.16. Let $(X, \tau)$ be an $A C D H-1$ space and let $F$ be a related finite set. If $x \neq y$ and $\{x, y\} \cap$ $F=\emptyset$ and $x \in \bar{y}$, then $\bar{x}$ is an infinite set.

Proof. Suppose that $x \neq y,\{x, y\} \cap F=\emptyset$ and $x \in \bar{y}$. Suppose that $\bar{x}$ is a finite set, then there is $N \in \mathbb{N}$ such that $|\bar{x}|=N$. Let $K$ be any countable dense set in $X$, then by Theorem 4.14, there is a countable collection of subsets of $K$ say $A_{1}, A_{2}, \ldots$ satisfing the conditions (i)-(vii). So, $A=\bigcup_{n=1}^{m} A_{n} \subseteq K$ and $\bar{A}=\bar{K}=X$. Let $I=\left\{i:\left|A_{i}\right|=\aleph_{0}\right\}$. For $i \in I$, define $B_{i}=$ $A_{i} \backslash\left\{a_{1}^{i}, a_{2}^{i}, \ldots, a_{N}^{i}\right\}$ and for $i \notin I B_{i}=A_{i}=\left\{a_{i}\right\}$, also define $B=\bigcup_{i=1}^{m} B_{i}$. To show that $\bar{B}=\bar{A}$. If $i \in I$, then $\mathrm{Cl}\left\{a_{1}^{k}, a_{2}^{k}, \ldots, a_{N}^{k}\right\} \subseteq \mathrm{Cl}\left\{a_{N+1}^{i}\right\} \subseteq \bar{B}_{i}$, therefore $\bar{A}_{i} \subseteq \bar{B}_{i} \cup \mathrm{Cl}\left\{a_{1}^{i}, a_{2}^{i}, \ldots, a_{N}^{i}\right\} \subseteq \bar{B}_{i}$. Then $B$ is a countable dense set in $X$ and so is $B \cup\{x, y\}$. Therefore, there exists $h \in H(X)$ such that $h((B \cup\{x, y\} \backslash F))=B \backslash F$. As $x \notin F$, we have $h(x) \in B_{i}$ for some $i \in \mathbb{N}$. If $\left|B_{i}\right|=1$, then $B_{i}=\left\{b^{i}\right\}$ and $h(x)=b^{i}$, so $b^{i} \in h(\bar{y})$ where $y \notin F$; therefore, $h(y) \in B \backslash\left\{b^{i}\right\}$ which is a contradiction by Theorem 4.14(vi). If $\left|B_{i}\right|=\aleph_{0}$ and $h(x)=b_{n_{o}}^{i}$ for some $n_{o}>N$, so all $b_{i}^{k}, b_{2}^{i}, \ldots, b_{n}^{i}$ are in $\overline{h(x)}$. As $|\overline{h(x)}|=|\bar{x}|>N$, which is impossible, so $\bar{x}$ is an infinite set.

Recall that in ACDH-1 space, if $K$ is a countable dense subset of $X$, then by Theorem 4.14 there are countable subsets $A_{1}, A_{2}, \ldots$ of $K$ satisfying (i)-(vii) of the pointed theorem.

Moreover, $A=\bigcup_{i=1}^{m} A_{i} \subseteq K, \bar{A}=\bar{K}=X$. Therefore, there is $h \in H(X)$ such that $h(A \backslash F)=K \backslash F$. By Proposition 4.15, $K \backslash F$ can be decomposed in the same way as $A \backslash F$.

The following theorem shows that all the above $A_{i}$ s are singletons.

Theorem 4.17. Let $(X, \tau)$ be an $A C D H-1$ space, $K$ any countable dense subset of $X$, and $F$ a related finite set. Then $K \backslash F=\bigcup_{i=1}^{\infty} A_{i}$ and $\left|A_{i}\right|=1$. 
Proof. Let $I=\left\{i:\left|A_{i}\right|=\aleph_{0}\right\}$. If $I \neq \emptyset$, then $A_{i}=\left\{a_{1}^{i}, a_{2}^{i}, \ldots\right\}$, for some $i \in I$. We have $a_{1}^{i} \in$ $\mathrm{Cl}\left\{a_{2}^{i}\right\}$ and $\left\{a_{1}^{i}, a_{2}^{i}\right\} \cap F=\emptyset$, so, by Theorem 4.16, we have $\mathrm{Cl}\left\{a_{1}^{i}\right\}$ which is an infinite set. Let $a_{0}^{i} \notin F$ with $a_{0}^{i} \in \operatorname{Cl}\left\{a_{1}^{i}\right\} \backslash\left\{a_{1}^{i}\right\}$. In a similar way, let $a_{-1}^{i} \notin F$ with $a_{-1}^{i} \in \operatorname{Cl}\left\{a_{0}^{i}\right\} \backslash\left\{a_{0}^{i}\right\}$. By the same argument, we have a sequence $\ldots, a_{-n}^{i} a_{-n+1}^{i}, \ldots, a_{-1}^{i}, a_{0}^{i}$ and $a_{-k}^{i} \in \operatorname{Cl}\left\{a_{-k+1}^{i}\right\} \backslash\left\{a_{-k+1}^{i}\right\}$. Now we claim that for all $k, n \in \mathbb{N}, a_{-k}^{i} \neq a_{n}^{i}$. If $a_{-k}^{i}=a_{n}^{i}$ for some $n \geq 2$ and $k \geq 0$, then $a_{n}^{i} \in \mathrm{Cl}\left\{a_{1}^{i}\right\}$ which contradicts the fact that $\left\{a_{1}^{i}, a_{2}^{i}, \ldots\right\}$ is a closurely ordered set. Also $a_{1}^{i} \neq a_{-k}^{i}$ for all $k>0$, since $a_{1}^{i} \notin \mathrm{Cl}\left\{a_{0}^{i}\right\}$ and $a_{-k}^{i} \in \mathrm{Cl}\left\{a_{0}^{i}\right\}$, so we proved our claim. Let $K_{1}=\left[\bigcup_{i \notin I} A_{i}\right] \cup$ $\left[\bigcup_{i \in I}\left\{\ldots, a_{-2}^{i} a_{-1}^{i}, a_{0}^{i}, a_{1}^{i}, a_{1}^{i}, \ldots\right\}\right]$. Then $X=\bar{K} \subseteq \overline{K_{1} \cup F}$, hence $K_{1} \cup F$ is a countable dense subset of $X$. Then there is $h \in H(X)$ such that $h(K \backslash F)=\left(K_{1} \cup F\right) \backslash F=K_{1}$. For $i \notin I, A_{i}=\left\{a^{i}\right\}$ and $a^{i} \notin \bar{x}$, for all $x \in(K \backslash F) \backslash\left\{a^{i}\right\}$. Then by Proposition 4.15, $h\left(A_{i}\right)=\left\{a^{j}\right\}=A_{j}, j \notin I$. Now define $i_{0}=\inf (I)$, therefore $A_{i_{0}}=\left\{a_{1}^{i_{0}}, a_{2}^{i_{0}}, \ldots\right\}$. Moreover, $h\left(a_{1}^{i_{0}}\right) \in A_{j_{0}}$, for some $j_{0} \in I$, where $A_{j_{0}}=\left\{\ldots, a_{-2}^{j_{0}}, a_{-1}^{j_{0}}, \ldots, a_{1}^{j_{0}}, a_{2}^{j_{0}}, \ldots\right\}$. Then $h\left(a_{1}^{i_{0}}\right)=a_{k}^{j_{0}}$, for some $k \in \mathbb{Z}$. Also $x \in \operatorname{Cl}\left\{a_{1}^{i_{0}}\right\}$, where $x \in K \backslash F$ and $x=h^{-1}\left(a_{k-1}^{j_{0}}\right)$. If $x \in A_{r}$, for some $r \in I$, then $A_{r} \cap \mathrm{Cl}\left\{\bigcup_{j=1}^{r-1} A_{j}\right\} \neq \emptyset$ which is a contradiction, so $I=\phi$.

As a consequence of the previous theorem, we have the following results.

Corollary 4.18. If $(X, \tau)$ is an ACDH-1 space, $F$ is a related finite set, and $K$ is a countable dense subset of $X$, then $K \backslash F$ has the closurely ordered property.

Proof. If $K$ is a countable dense subset of $X$, then by using Theorem 4.17, we have that $K \backslash F=$ $\bigcup_{i=1}^{\infty} A_{i}$ and $\left|A_{i}\right|=1$, for all $i \in \mathbb{N}$. Therefore, $K \backslash F=\bigcup_{i=1}^{\infty} a^{i}$ indeed, $a^{i} \notin \operatorname{Cl}\left\{a^{j}\right\}$, for all $i \neq j$.

Corollary 4.19. Every $A C D H-1$ space is an $A S T_{1}$ space.

Proof. Let $F$ be a related finite set and $x \neq y$ with $\{x, y\} \cap F=\emptyset$. If $K$ is a countable dense subset of $X$, say $K=\left\{x_{1}, x_{2}, \ldots\right\}$, then the set $K_{1}=\left\{x, y, x_{1}, x_{2}, \ldots\right\}$ is also a countable dense subset of $X$, therefore by Corollary 4.18 , we have that $K_{1} \backslash F$ has the closurely ordered property and $\{x, y\} \subseteq K_{1} \backslash F$, therefore $y \notin \bar{x}$. Similarly, $x \notin \bar{y}$.

\section{Acknowledgment}

This paper is financially supported by the Deanship of Academic Research at the University of Jordan, Amman, Jordan.

\section{References}

[1] W. Sierpinski, "Sur une propiete topologique des ensembles denombrable dense en Soi," Fundamenta Mathematicae, vol. 1, pp. 11-28, 1920.

[2] M. Fréchet, "Les dimensions d'un ensemble abstrait," Mathematische Annalen, vol. 68, no. 2, pp. 145$168,1910$.

[3] L. Brouwer, "Some remarks on the coherence type n," Proceedings Akadamie van Wetenschappen Amsterdam, vol. 15, no. 2, pp. 1256-1263, 1913.

[4] R. Bennett, “Countable dense homogeneous spaces," Fundamenta Mathematicae, vol. 74, no. 3, pp. 189194, 1972.

[5] N. Lauer, Countable dense homogeneous spaces and densely homogeneous spaces, Ph.D. thesis, Auburn University, Auburn, Ala, USA, 1974.

[6] B. Fitzpatrick Jr., J. M. S. White, and H. X. Zhou, "Homogeneity and $\sigma$-discrete sets," Topology and Its Applications, vol. 44, no. 1-3, pp. 143-147, 1992. 
[7] A. Tallafha, A. Al-Bsoul, and A. Fora, "Closurely ordered countable sets and applications," Global Journal of Pure and Applied Mathematics, vol. 1, no. 2, pp. 177-182, 2005.

[8] A. Fora, A. Tallafha, and A. Al-Bsoul, "Almost countable dense homogeneous spaces," to appear. 


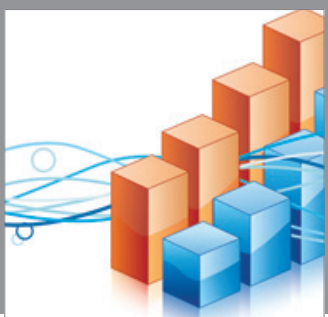

Advances in

Operations Research

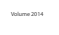

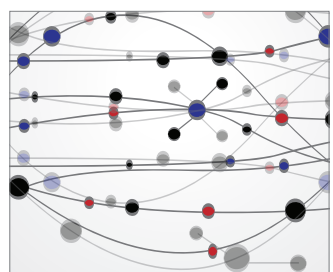

\section{The Scientific} World Journal
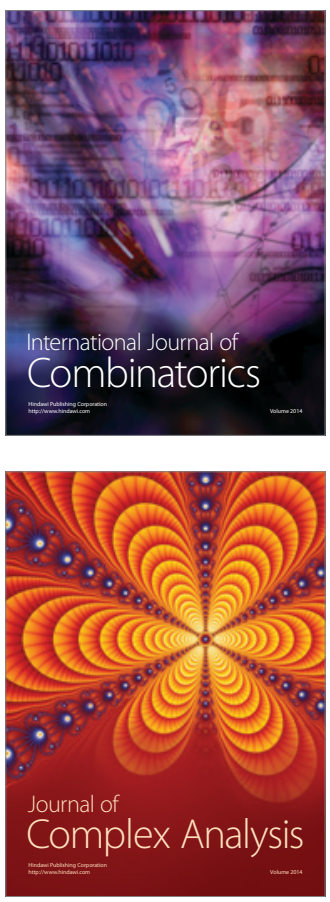

International Journal of

Mathematics and

Mathematical

Sciences
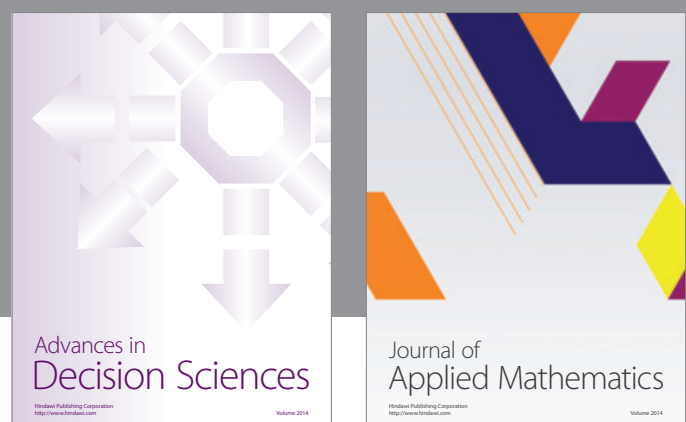

Journal of

Applied Mathematics
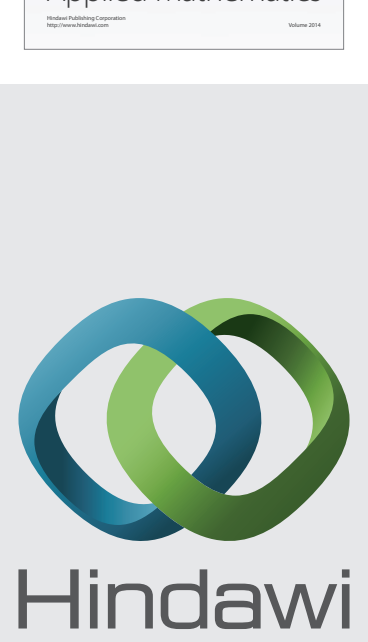

Submit your manuscripts at http://www.hindawi.com
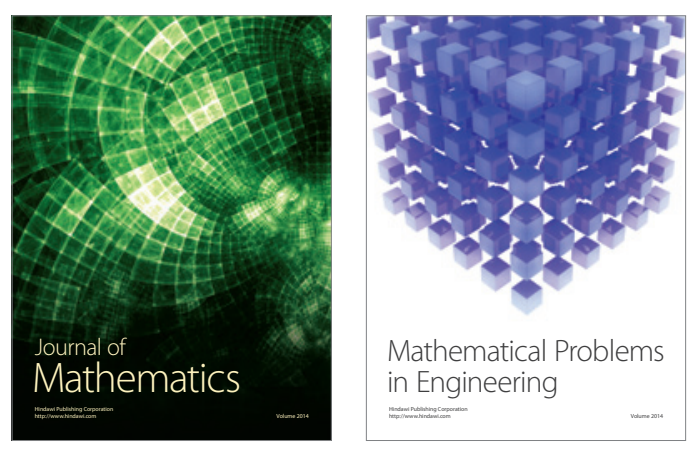

Mathematical Problems in Engineering
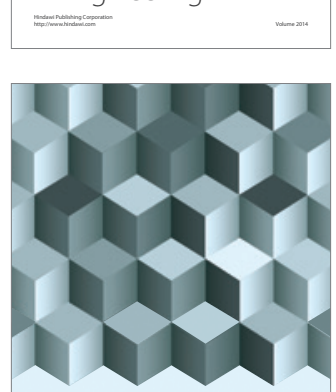

Journal of

Function Spaces
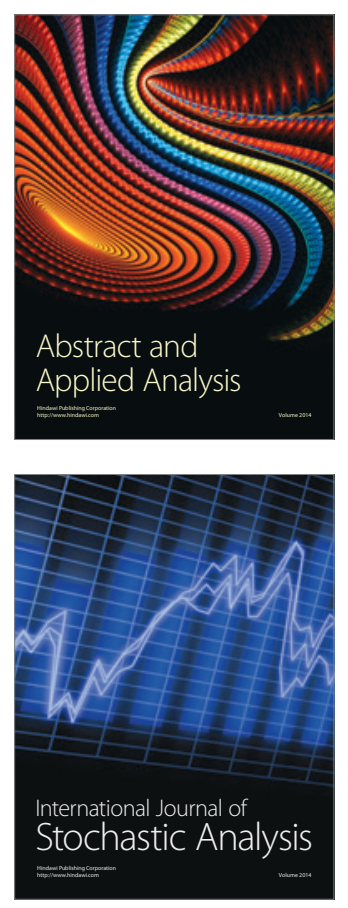

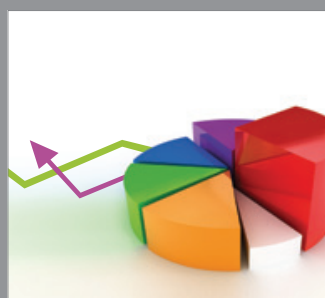

ournal of

Probability and Statistics

Promensencen
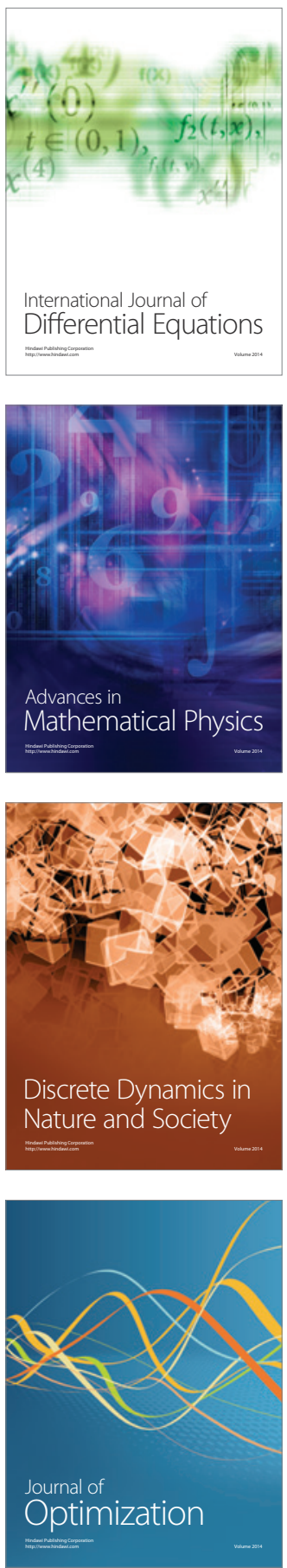